\title{
A case of synchronous intramucosal gastric carcinoma with multiple lymph node metastases
}

\author{
En Amada ${ }^{1}$, Hirofumi Kawakubo ${ }^{1 *}$, Satoru Matsuda', Shuhei Mayanagi ${ }^{1}$, Rieko Nakamura ${ }^{1}$, Tomoyuki Irino ${ }^{1}$, \\ Norihito Wada', Shuji Mikami² and Yuko Kitagawa' ${ }^{1}$
}

\begin{abstract}
Background: In Japan, the prevalence of synchronous multiple intramucosal gastric carcinoma is reported to be $5-15 \%$. Here is a case of a synchronous small gastric carcinoma fulfilling the definite indication and curative criteria for endoscopic submucosal dissection with multiple lymph node metastases.

Case presentation: A Japanese woman in her fifties with a history of endoscopic resection for mucosal poorly differentiated adenocarcinoma was evaluated, with the UICC TNM classification stage being CT1aNOMO cStagelA. She had undergone total gastrectomy with D1 + lymph node dissection. Histopathological examination revealed 16 individual sporadic lesions in the gastric body, with maximum diameter $3 \mathrm{~mm}$ and localization in the lamina propria. Twentyseven nodes were resected, and metastasis of the carcinoma was revealed in 24 nodes.

Conclusions: Undifferentiated intramucosal gastric cancer has a relatively high probability of lymph node metastasis; however, synchronous early lesions are often overlooked. Frequent follow-up examinations may increase the detection of multiple gastric cancers.
\end{abstract}

Keywords: Stomach neoplasms, Multiple primary neoplasms, Lymphatic metastasis

\section{Introduction}

In Japan, the prevalence of synchronous multiple intramucosal gastric carcinoma is reported to be $5-15 \%$. It relatively frequently occurred in elderly males and in patients with adenoma, atrophic gastritis, or a family history of gastric cancer compared to patients with solitary early gastric cancer [1]. We experienced a case of early gastric carcinoma in middle-aged female that was widely scattered troughout the gastric body, each lesion of which had a definite indication for curative resection of ER based on the Gastric Cancer Treatment Guidelines' (ver. 4) criteria at that time [2]; this case was concluded to have multiple lymph node metastases.

\footnotetext{
*Correspondence: hkawakubo@keio.jp

${ }^{1}$ Department of Surgery, Keio University School of Medicine, 35

Shinanomachi, Shinjuku, Tokyo, Japan

Full list of author information is available at the end of the article
}

\section{Case presentation}

A Japanese woman in her fifties presented at a clinic with dysphagia. She had no family history of cancer. Upper gastrointestinal endoscope (EGD) had been performed, and two reddened lesions $20 \mathrm{~mm}$ in size were observed at the greater curvature of the middle-third and lower-third of the gastric body. A biopsy was taken from one of the lesions and revealed signet-ring cell carcinoma. She was referred to our institution for further treatment. Endoscopic submucosal dissection (ESD) had been performed on these two lesions. The histopathological examination revealed that both lesions were poorly differentiated adenocarcinoma, which was localized in the lamina propria with no lymphatic or vascular invasion. Endoscopists at our institute performed follow-up-EGD performed 4, 7, 8 and 9 months after the ESD, since the initial EGD performed at our institute revealed multiple reddened 
area with no findings that mentions existence of malignancy at that time. And EGD performed on 9 months after the ESD showed that more than ten small flat erosive lesions less than $5 \mathrm{~mm}$ in diameter were widely scattered throughout the middle-third and lower-third of the gastric body (Fig. 1). Magnified narrow-band imaging revealed that all the lesions had a corkscrew-like irregular microvascular pattern with a normal glandular structure, which was presumed to be the existence of poorly differentiated carcinoma cells. A biopsy specimen

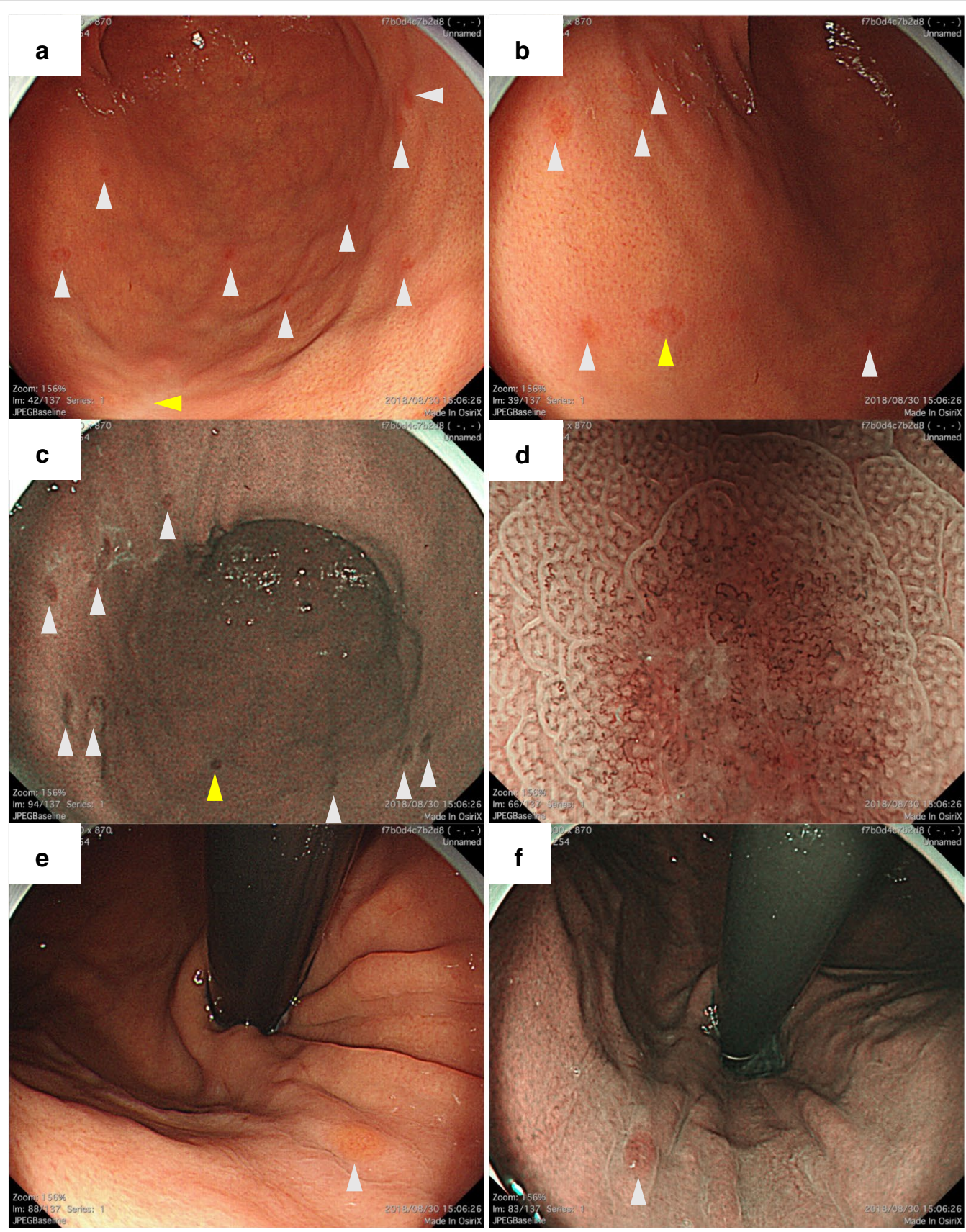

Fig. 1 Endoscopic image in the preoperative state. $\mathbf{a}$ and $\mathbf{b}$ : White light images of the middle-third and lower-third of the gastric body. More than ten small flat erosive lesions less than $5 \mathrm{~mm}$ in diameter were widely scattered (white and yellow arrowheads). By narrow-band imaging (c), those lesions were observed as brownish lesions (white and yellow arrowheads). $\mathbf{d}$ A magnified image of the lesion identified by a yellow arrowhead. A corkscrew-like irregular microvascular pattern with normal grandular structure was observed. e and $\mathbf{f}$ : White light and narrow-band images of lesions located at the upper gastric body 
was obtained from the lesion at the posterior wall of the lower-third of the gastric body. The specimen contained Periodic Acid-Schiff staining positive, pan-cytokeratin (clone: AE1/AE3) positive, and p53 weakly positive cells and was demonstrated to be poorly differentiated adenocarcinoma. Contrasted computed tomography imaging showed no distant metastasis and no swelling of the regional lymph nodes (Fig. 2). Furthermore, Positron Emission Tomography imaging showed no abnormal accumulation of 18F-fluorodeoxyglucose in her body. The physical examination and routine laboratory examination results were unremarkable, including carcinoembryonic antigen (CEA) and carbohydrate antigen (CA) 19-9, which were within normal limits $(1.1 \mathrm{ng} / \mathrm{ml}$ and $20 \mathrm{U} / \mathrm{ml}$, respectively). Serum Helicobacter-pylori-antibody test and pepsinogen test were negative. The UICC TNM classification staging for her gastric cancer was cT1aN0M0 cStage IA, and each lesion had a definitive indication for ESD; however, the lesions were diffusely located along the entire gastric wall, and ESD was expected to create a large defect of the gastric mucosa. The case was

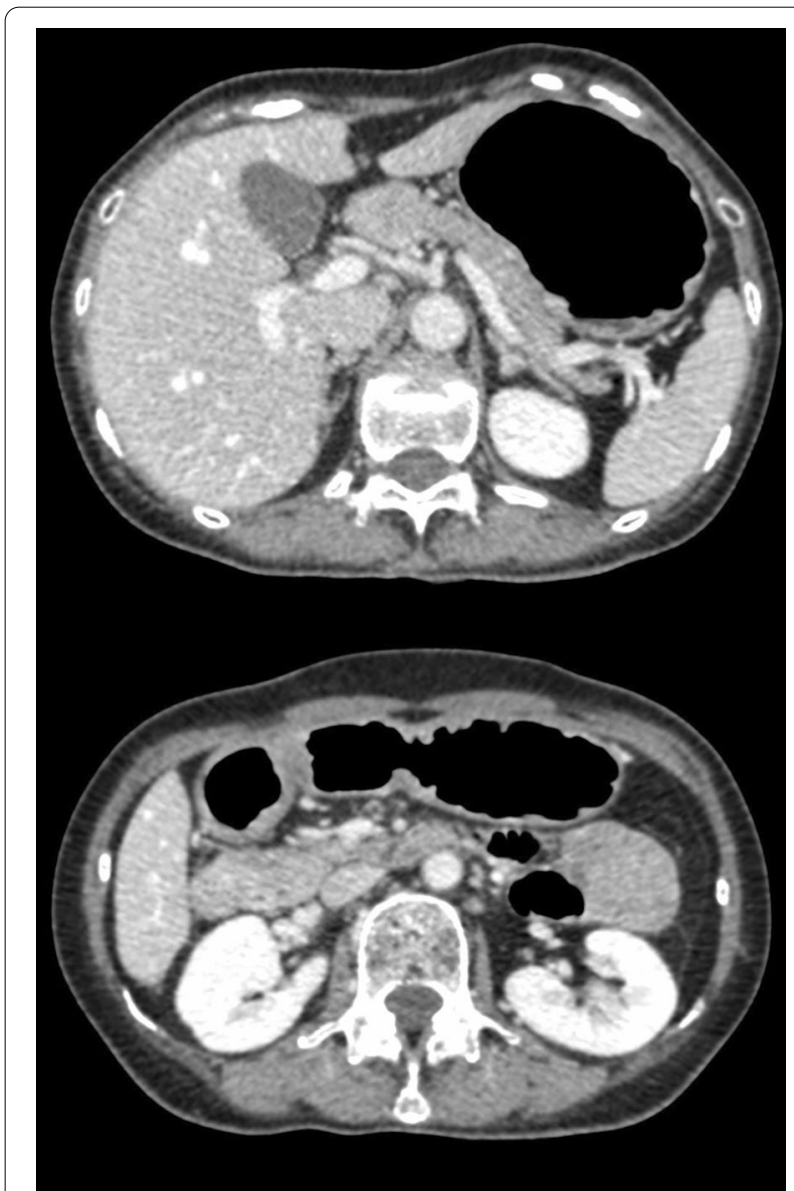

Fig. 2 Contrast computed tomography image showed no swelling lymph nodes or distantmetastasis meticulously discussed by a conference of experts, and under full consent of the patient, a total gastrectomy was planned. She underwent robot-assisted total gastrectomy with D1+lymph node dissection. She was discharged from the hospital on the 8th postoperative day. The final diagnosis was pT1aN3bM0 pStage IIIB, and she began adjuvant chemotherapy with tegafur, gimeracil, oteracil, and oxaliplatin. She is confirmed to be alive with no recurrence at 8 months after the operation (Fig. 3).

\section{Histopathological findings}

Sixteen individual sporadic lesions were observed in the gastric body. The lesions were a maximum of $3 \mathrm{~mm}$ in diameter. In each lesion, atypical cells with hyperchromatic nuclei were proliferating in the lamina propria. Each lesion contained Periodic Acid-Schiff staining positive, pan-cytokeratin (clone: AE1/AE3) positive, p53 weakly positive, Chromogranin A negative, and a -fetoprotein negative cells and was demonstrated to be poorly differentiated adenocarcinoma. No lymphatic or vascular invasion was observed. A total of 27 lymph nodes had been resected, and metastasis of the carcinoma was revealed in 24 nodes. The following lymph node stations displayed metastasis of the carcinoma: \#1, \#3b, \#4d,

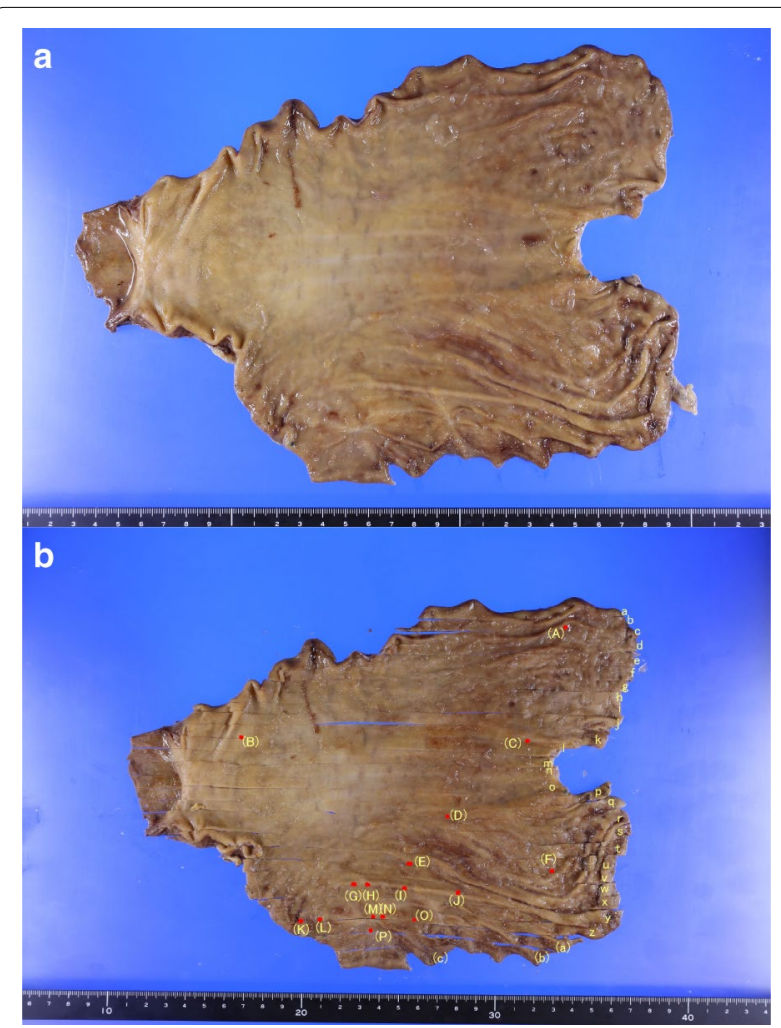

Fig. 3 Macroscopic image of the resected specimen. a Original formalin-fixed specimen. $\mathbf{b}$ Mapping of multiple lesions on the gastric body. Sixteen individual sporadic lesions were observed 
$\# 5$, \#6, \#9, and \#11p (the lymph-node station number is based on the Japanese classification of gastric carcinoma: 3rd English edition [3]. The number of resected lymph nodes and metastatic lymph nodes were as follows: \#1 1/1, \#3 1/1, \#4d 1/1, \#5 2/3, \#6 6/6, \#9 5/5, and \#11p 2/2 (metastatic/resected), respectively. Molecular biological analysis of resected specimen was tried; however, the amount of tumor tissue was small and it failed.

\section{Discussion}

ER has become the standard treatment for intramucosal gastric cancer with virtually no risk of LN metastasis because of its minimal invasiveness. The probability of lymph node metastasis among intramucosal gastric cancer is reported to be $1.2-2.2 \%$. However, undifferentiated intramucosal gastric cancer is reported to have a higher probability of LN metastasis (4.2\%) [4]. Seung-Young [5] has reported that undifferentiated-type cancers, including poorly differentiated adenocarcinoma, signet-ring cell carcinoma, poorly cohesive carcinoma, and mucinous adenocarcinoma, are associated with increased risks of LN metastasis (odds ratio 6.104, 95\% CI 1.317-28.284, $p=0.0021)$. Among undifferentiated gastric carcinomas, female sex, younger age, larger tumor size $(>20 \mathrm{~mm}$ in diameter), the presence of ulcers, and the presence of lymphovascular invasion are considered risk factors for lymph node metastasis [6]. In most reported cases, the metastatic lymph node was localized in the D1+ regional lymph nodes. Furthermore, for synchronous gastric neoplasms, synchronous lesions were reported to have a flat and depressed macroscopic appearance [7]. The absence of Helicobacter pylori infection, lower third location, and the presence of intestinal metaplasia were risk factors for multiple neoplasms [8]. We performed total gastrectomy for synchronous intramucosal gastric cancer all of which fulfilled indication criteria of ER. Seo et al. reported metachronous early gastric malignant lesions had significantly high incidence of containing undifferentiated cancer cells [7]. And all patients who developed metachronous lesions were discovered within 1 year after endoscopic resection. In addition, Fujita et al. reported patients that underwent gastrectomy with combination of the histological differentiation of the main and sublesion of the initial cancers were undifferentiated type had a higher incidence of subsequent remnant gastric cancer [9]. These reports imply that total gastrectomy is supposed to be one option for sporadic early gastric cancer (Figs. 4 and 5).

Existence of specific genetic characteristic is suggested in sporadic intramucosal gastric cancer. Kim et al. reported synchronous gastric neoplasms are correlate with high microsatellite instability (MSI-high) [10]. Patients with MSI-high gastric cancer showed higher prevalence of gastric adenoma than those with microsatellite stable gastric cancers. In addition, MSI-high gastric adenomas, loss-of-functional events of APC is frequently observed, and it is considered to initiates the gastric carcinogenesis and is followed by mutations of histone modifiers and then activation of cancer-related genes [11]. Moreover, Zazula et al. reported gastric cancer with the MSI-high phenotype revealed CDH1 promoter hypermethylation, of which mutation is frequently observed in hereditary diffuse gastric cancer patients [12]. According to The Cancer Genome Atlas project, MSI-high gastric cancer is reported to consist $21.7 \%$ of all gastric cancer [13]. In addition, MSI-high gastric cancer showed hypermethylation at the MLH1 promoter, one of the mismatch repair genes. In addition, sporadic signet-ring cell gastric cancer is reported to be one of the key features of gastric cancer triggered by CDH1 mutation [14]. The germline mutation of $\mathrm{CDH} 1$ is inherited by autosomal dominant manner and develops Hereditary Diffuse Gastric Cancer (HDGC). The diagnostic criteria of HDGC by International Gastric Cancer Linkage Consortium include: (i) two or more gastric cancer cases in the family, with one being a confirmed diffuse gastric cancer diagnosed before the age of 50 years; (ii) three or more confirmed diffuse gastric cancers in first- or second-degree relatives, independent of age; and (iii) diffuse gastric cancer diagnosed before the age of 40 years without additional family history [15]. In addition, CDH1 testing could be considered in patients with bilateral or familial LBC before the age of 50, patients with DGC and cleft lip/palate, and those with precursor lesions for signet ring cell carcinoma. The clinical feature of this case implies the existence of CDH1 mutation; however, the family history suggests that the mutation might have occurred de novo in this precise case. As for Japanese, only one case of diffuse gastric cancer associated with a de novo genomic deletion of CDH1 gene has been reported so far [16].

\section{Conclusion}

We experienced a synchronous small gastric carcinoma without ulceration and lymphovascular invasion of a relatively young female that emerged after initial ESD. Synchronous lesions are reported to be overlooked in $32.4 \%$ of early gastric neoplasia cases [17]; however, the prognosis of small mucosal gastric cancer is favorable, with a reported 5-year relative postoperative survival of $90 \%$ or more [18]. As for patients with synchronous early gastric carcinoma, detection frequency of massively invading cancer detected more than in 1 year was reported to be less than 1\% [19]. Based on these findings, annual surveillance by EGD is recommended to be performed. 

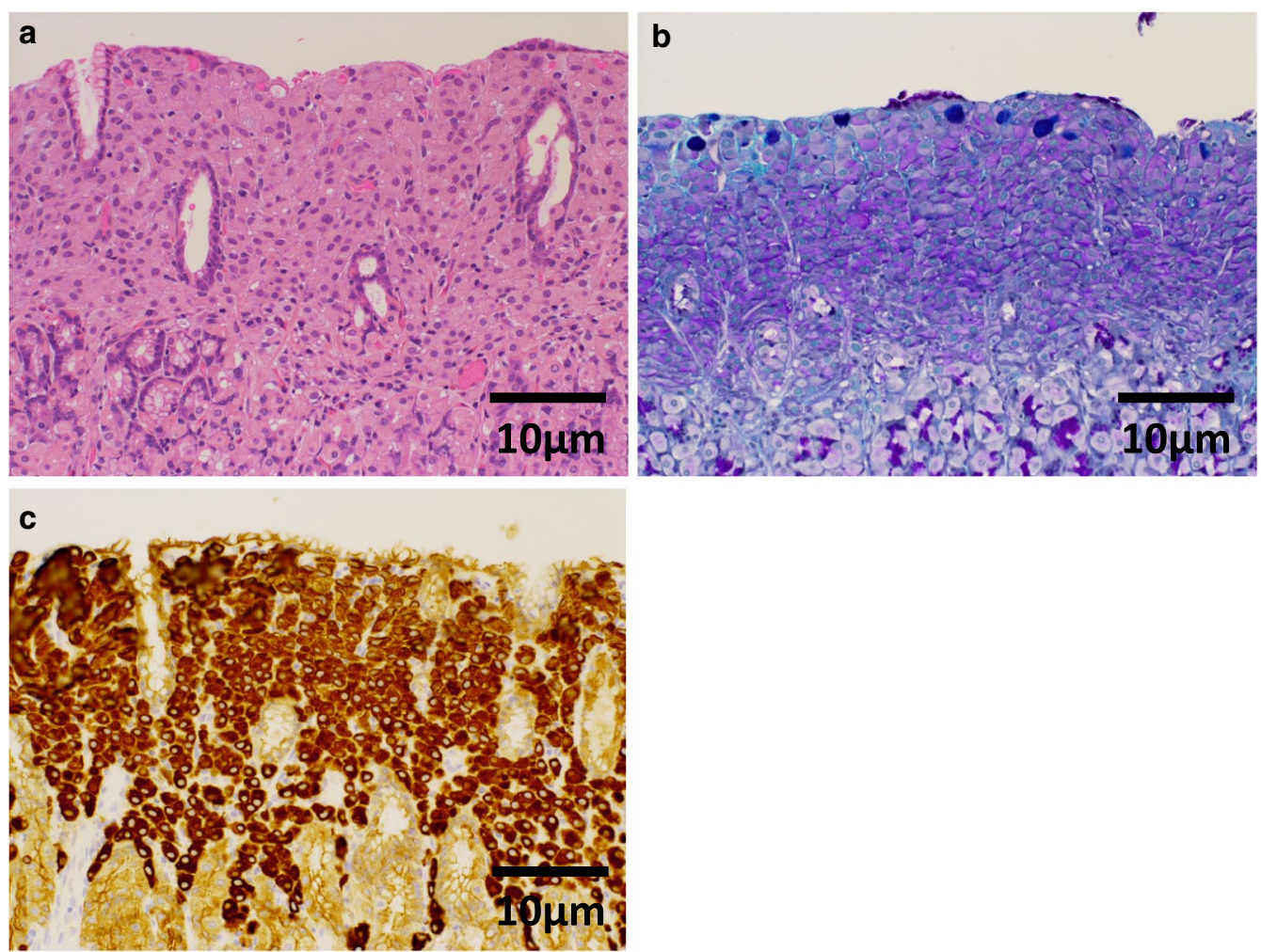

Fig. 4 Microscopic image of lesion (O) at Fig. 3. a Hematoxylin-eosin (HE) staining, b Periodic Acid-Schiff (PAS) and alcian blue staining, and c immunostaining of pan-cytokeratin (clone: AE1/AE3). HE staining shows that atypical cells with hyperchromatic nuclei were proliferating in the lamina propria. The lesion contains PAS staining positive, pan-cytokeratin (clone: AE1/AE3) positive cells. No lymphatic or vascular invasion was observed
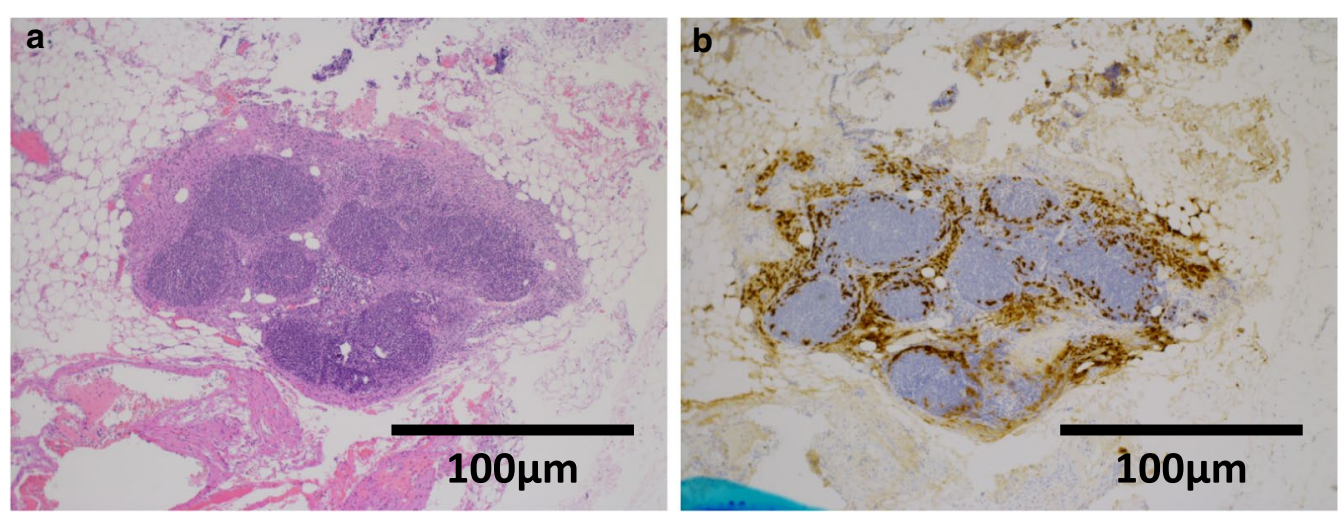

Fig. 5 Microscopic image of metastatic lymph node station No. 5. a HE staining and $\mathbf{b}$ immunostaining of pan-cytokeratin (clone: AE1/AE3). Cancer cells are described as pan-cytokeratin (clone: AE1/AE3)

\section{Abbreviations}

GCTGs: The Gastric Cancer Treatment Guidelines; ER: Endoscopic resection; EGD: Upper gastrointestinal endoscope; ESD: Endoscopic submucosal dissection; CEA: Carcinoembryonic antigen; CA: Carbohydrate antigen; MSI: Microsatellite instability; APC: Adenomatous polyposis coli; $\mathrm{CDH1}$ : E-cadherin; MLH1: Mismatch repair protein; HDGC: Hereditary diffuse gastric cancer; DGC: Diffuse gastric cancers; LBC: Lobular breast cancer.

\section{Acknowledgements}

None.

\section{Authors' contributions}

EA is the first author and prepared the manuscript under the supervision of HK. HK and EA performed the operation. The authors read and approved the final manuscript. SM, SM, RM, TI and NW contributed to the surgical 
management of the patient and supported the preparation of the manuscript. YK revised the manuscript. SM and KK performed and interpreted the pathological diagnosis of the resected specimen. All authors read and approved the final manuscript.

\section{Funding}

The authors received no specific funding for this work.

\section{Availability of data and materials}

All data generated or analyzed during this study are included in this published article. We would not like to share data other than those described in the paper, because they include personal information.

\section{Ethics approval and consent to participate}

The present study was conducted in accordance with the ethical standards of our institution.

\section{Consent for publication}

When obtaining informed consent for admission and the surgical procedure, general consent was also obtained from the patient for publication and presentation.

\section{Competing interests}

The authors declare that they have no competing interests regarding this manuscript.

\section{Author details}

${ }^{1}$ Department of Surgery, Keio University School of Medicine, 35 Shinanomachi, Shinjuku, Tokyo, Japan. ${ }^{2}$ Division of Diagnostic Pathology, Keio University School of Medicine, 35 Shinanomachi, Shinjuku, Tokyo, Japan.

Received: 10 June 2020 Accepted: 2 March 2021

Published online: 02 April 2021

\section{References}

1. Lee IS, Park YS, Kim KC, Kim TH, Kim HS, Choi KD, et al. Multiple synchronous early gastric cancers: high-risk group and proper management. Surg Oncol. 2012;21(4):269-73.

2. Japanese Gastric Cancer A. Japanese gastric cancer treatment guidelines 2014 (ver. 4). Gastric Cancer. 2017;20:1-19.

3. Japanese Gastric Cancer Association. Japanese classification of gastric carcinoma: 3rd English edition. Gastric Cancer. 2011;14:101-12.

4. Gotoda T, Yanagisawa A, Sasako M, Ono H, Nakanishi Y, Shimoda T, et al. Incidence of lymph node metastasis from early gastric cancer: estimation with a large number of cases at two large centers. Gastric Cancer. 2000;3:219-25.

5. Oh SY, Lee KG, Suh YS, Kim MA, Kong SH, Lee HJ, et al. Lymph node metastasis in mucosal gastric cancer: reappraisal of expanded indication of endoscopic submucosal dissection. Ann Surg. 2017;265:137-42.

6. Lee JH, Choi IJ, Han HS, Kim YW, Ryu KW, Yoon HM, et al. Risk of lymph node metastasis in differentiated type mucosal early gastric cancer mixed with minor undifferentiated type histology. Ann Surg Oncol. 2015;22:1813-9.

7. Seo JH, Park JC, Kim YJ, Shin SK, Lee YC, Lee SK. Undifferentiated histology after endoscopic resection may predict synchronous and metachronous occurrence of early gastric cancer. Digestion. 2010;81:35-42.

8. Lim JH, Kim SG, Choi J, Im JP, Kim JS, Jung HC. Risk factors for synchronous or metachronous tumor development after endoscopic resection of gastric neoplasms. Gastric Cancer. 2015;18:817-23.

9. Fujita T, Gotohda N, Takahashi S, Nakagohri T, Konishi M, Kinoshita T. Relationship between the histological type of initial lesions and the risk for the development of remnant gastric cancers after gastrectomy for synchronous multiple gastric cancers. World J Surg. 2010;34:296-302.

10. Kim YB, Lee SY, Kim JH, Sung IK, Park HS, Shim CS, et al. Microsatellite instability of gastric and colorectal cancers as a predictor of synchronous gastric or colorectal neoplasms. Gut Liver. 2016;10:220-7.

11. Lim C-H, Cho YK, Kim SW, Choi M-G, Rhee J-K, Chung Y-J, et al. The chronological sequence of somatic mutations in early gastric carcinogenesis inferred from multiregion sequencing of gastric adenomas. Oncotarget. 2016;7:39758-67.

12. Zazula M, Ferreira AM, Czopek JP, Kolodziejczyk P, Sinczak-Kuta A, Klimkowska $\mathrm{A}$, et al. $\mathrm{CDH} 1$ gene promoter hypermethylation in gastric cancer: relationship to Goseki grading, microsatellite instability status, and EBV invasion. Diagn Mol Pathol. 2006;15:24-9.

13. Cancer Genome Atlas Research N. Comprehensive molecular characterization of gastric adenocarcinoma. Nature. 2014;513:202-9.

14. Masayoshi Yamada M, Takeo Fukagawa T, Takeshi Nakajima T, Kiyoshi Asada K, Shigeki Sekine S, Satoshi Yamashita S, et al. Hereditary diffuse gastric cancer in a Japanese family with a large deletion involving $\mathrm{CDH}$ 1. Gastric Cancer. 2014;17:750-6.

15. van der Post RS, Vogelaar IP, Carneiro F, Guilford P, Huntsman D, Hoogerbrugge $\mathrm{N}$, et al. Hereditary diffuse gastric cancer: updated clinical guidelines with an emphasis on germline $\mathrm{CDH} 1$ mutation carriers. J Med Genet. 2015;52:361-74.

16. Sugimoto S, Yamada H, Takahashi M, Morohoshi Y, Yamaguchi N, Tsunoda $Y$, et al. Early-onset diffuse gastric cancer associated with a de novo large genomic deletion of CDH1 gene. Gastric Cancer. 2014;17:745-9.

17. Nam HS, Kim HW, Choi CW, Kang DH, Park SB, Kim SJ, et al. Characteristics of overlooked synchronous gastric epithelial neoplasia after endoscopic submucosal dissection. Medicine (Baltimore). 2018;97:e12536.

18. Takekoshi T, Baba Y, Ota H, Kato Y, Yanagisawa A, Takagi K, et al. Endoscopic resection of early gastriccarcinoma: results of a retrospective analysis of 308 cases. Endoscopy. 1994;26:352-8.

19. Kato M, Nishida T, Yamamoto K, Hayashi S, Kitamura S, Yabuta T, et al. Scheduled endoscopic surveillance controls secondary cancer after curative endoscopic resection for early gastric cancer: a multicentre retrospective cohort study by Osaka University ESD study group. Gut. 2013;62(10):1425-32.

\section{Publisher's Note}

Springer Nature remains neutral with regard to jurisdictional claims in published maps and institutional affiliations.

\section{Submit your manuscript to a SpringerOpen ${ }^{\odot}$ journal and benefit from:}

- Convenient online submission

- Rigorous peer review

- Open access: articles freely available online

- High visibility within the field

- Retaining the copyright to your article

Submit your next manuscript at $\boldsymbol{\nabla}$ springeropen.com 